\title{
Zero Pronoun Resolution in Japanese Discourse Based on Centering Theory
}

\author{
OKUMURA Manabu \\ School of Information Science, \\ Japan Advanced Institute of \\ Science and 'Technology \\ Tatsunokuchi, Ishikawa 923-12, Japan \\ oku@jaist.ac.jp \\ TAMURA Kouji \\ Department of Software Development, \\ Fujitisu \\ 1.015 Kamikodanaka, Nakahara-ku, \\ Kawasaki 211, Japan \\ k-tamura@candy.paso.fujitsu.co.jp
}

\begin{abstract}
Recently there have becn a number of works that model the zero pronom resolution with the concept called 'conter.' However, the usefulness of the previous centering frameworks has not fully cvaluated with naturally occurring discourses. furthermore, the previous centering theory has handled only the phenomena in successive simple sentences and has not: adequately addressed the way to handle complex sentences that are prevalent in naturally occurring discomrses. In this paper, wo present a method to handle complex sentences with the centering theory and describe our framework that identifies the antecedents of zero pro. nouns in naturally occuming Jajomese discourses. We also present the evaluation of our framework with real discourses.
\end{abstract}

\section{Introduction}

In many natural languages, clements that can be easily deduced by the reader aro frequently omitted from the expressions in discourses. Particalarly in Japanese discourses, this omission occurs more frequently and a zero pronom is often used to avoid repoating a noun phrase that appeared in the previous sentences. A zero pronom can be considered as a noun phrase which is of an obligatory case and which is not expressed but can be nuderstood through the context(Yoshimoto, 1986). Therefore, to understand a Japanese discourse, it is inportant, to identify the antecedents of zero pronouns.

Recently there have been a number of works that morlel the (zoro) pronoun resolntion with the concept called 'center'(Gros\% et al., 1995; Brennan et al., 1987; Walker et al., 1994; Kameyama, 1986). The centering, theory tries to islentify the antecedent of a (zero) pronoun by the idea that the contity that a sentence most centrally concerms(center) tends to bo expressed by a (zero) pronom. The centering theory has the following advantages. Because it uses only the surface information in sentences and does not need a huge amount of common sense knowledge to resolve (zero) pronoums, it is easy to implement, it on computer systems. Secondly, it can be appliexl to many languages(Cross ot al., 1995; Walker ot al., 1994).

In spite of these advantages, unfortmately, the usefulness of the previous centering francworks has not fully tested bocause only a small number of constructed discourses have been used for evaluation. We think they should be tested with a corpus of naturally occurring discourses. However, such a test is now difficult because the previons centering theory has only handled the phenomena in successive simple sentences and has not adecluately adclessed the way to handle complex sentemes that are prevalent, in naturally occurring discomises.

In this paper, we present a method to handle complex sentences with the centering theory and describe our framework that identifies the antecedents of zero pronoms in naturally occurring Japanese discourses. We also present the evaluation of our framework with real discounses.

In section two we ontline two versions of the centering theory that have been applied to Japanese zero pronoun resolution. In section there we explain how zero pronouns in complex sen tences can be handled based on the centering the ory. In section four: wo describe a set, of the experinnents that our zero pronoun resolution method in section three is applied to real Japanesse dis courses.

\section{2 'Two Versions of the Centering Theory}

In the centering theory, each sentence has two structures associated with it: a set of discourse entitics called forward-looking centers, $C_{f} s$, that appear in the sentence, and a special nomber of $C_{f}$ s called the backward-looking center, $C_{b}$. 'The: $C_{6}$ is the rliscourse entity that the sentence most centrally concens. $\Lambda C_{f}$ may becomo a $C_{b}$ later 
in the discourse. The set of $C_{f} \mathrm{~s}$ is ordered by their grammatical properties which are considered to reflect their degrees of salience. The centering theory specifies the following (heuristic) rule:

If the $C_{b}$ of the current sentence is the same as the $C_{b}$ of the previous sentence, a (zero) pronoun should be used.

There are two versions of the centering theory that have been applied to Japanese zero pronoun resolution: Kameyama's(Kameyama, 1986) and Walker's(Walker et al., 1994). Roughly both versions use the following same forward center ranking for Japanese:

$$
\begin{gathered}
\text { Topic }>\text { Empathy }>\text { Subject }>\text { Object } 2 \\
>\text { Object }>\text { Others, }
\end{gathered}
$$

where Empathy is a grammatical property that indicates the speaker's position in describing a situation. In addition to the above rule, Kameyama's version uses the property sharing constraint that two zero pronouns in adjacent sentences, which co-specify the same $C_{b}$, should share one of the grammatical properties. This constraint is used for ranking discourse entities in the order of prefcrence as the antecedent of a zero pronoun.

Walker's version, on the other hand, uses the following additional rules and constraint:

- Constraint

For each sentence $U_{i}$ :

The center, $C_{b}\left(U_{i}\right)$, is the highestranked element of $C_{f}\left(U_{i-1}\right)$ that appears in $U_{i}$.

- Rules

For each sentence $U_{i}$ :

1. If a certain element of $C_{f}\left(U_{i-1}\right)$ appears as a (zero) pronoun in $U_{i}$, then so is $C_{b}\left(U_{i}\right)$

2. Transition states are ordered, where the transition state is determined based on two factors: whether $C_{b}$ of the current sentence is the same as of the previous sentence, and whether $C_{b}$ is the same as the highest-ranked member of $C_{f}$ of the current sentence. This transition ordering is used for ranking discourse entities in the order of preference as the antecedent of a zoro pronoun.

Basically, when the centering algorithm is used for the (zero) pronoun resolution, the algorithm first generates all possible antecedents for (zero) pronouns in a sentence by enumerating all possible $C_{b}$ and $C_{f}$ pairs for the sentence, and then filters and ranks these possible antecedents with the constraint and rules that are mentioned above. The $C_{b}$ of the sentence is computed as the side effect of performing the (zero) pronoun resolution.

\section{Processing Complex Sentences with the Centering Theory}

In the centering theory that we outlined in the last section, 'sentence', that is its basic unit of processing, means the simple sentence that contains only one predicate(verb). The centering theory, therefore, has not adequately addressed the way to handle complex sentences that contain multiplo verbs. However, it is necessary to handle complex sentences that are prevalent in naturally occurring discourses with the centering algorithms.

We can think of (at least) two ways to handle complex sentences. For instance, consider processing a complex sentence of the form 'SX Conj SY,' where SX and SY each consists of a simple sentence and Conj is a conjunctive element(Suri and $\mathrm{McCoy}, 1994)^{1}$. One can imagine processing SX first and then SY as if they are a lincar sequence of simple sentences and applying the centering theory to each sentence successively and updating the data structures for centering.

On the other hand, the whole sentence can be treated as a single unit. This approach, however, has two problems. First, the intrasentential ellipsis that the antecedent exists in the same sentence ${ }^{2}$ cannot be handled with the centering theory, because the centering theory only handles the intersentential ellipsis. Therefore, the intrasentential ellipsis must be dealt with separately from the intersentential ellipsis. Secondly, in the centering theory, it is unclear whether two zero pronouns with the same grammatical property in the different simple sentences (of a complex sentence) can be simultaneously handled without any extension to the original theory.

Comparing these two approaches, we adopt the former. And if a sentence contains multiple verbs, we partition it into multiple simple sentencos and apply the centering theory to a sequence of partitioned simple sentences individually for the zero pronoun resolution. Using this approach, we need not modify the original centering algorithm drastically to handle complex sentences. Even the intrasentential ellipsis can be handled with the contering theory, because different simple sentences contain the antecedent and the zero pronoun respectively, after partitioning.

\subsection{The range of search for the antecedent}

Since the contering theory uses only the information in the previous and current sentences, this might be problematic whon we adopt the 'partition' approach. For example, if the previous sentence consists of three simple sentences,

\footnotetext{
${ }^{1}$ In case of Japanese, it is a conjunctive postposition.

${ }^{2}$ Of course, the antecedent does not exist in the same simple sentence.
} 
the first simple sentence in the previous sentence becomes the third from the current sentence, after partitioning. l'artitioning might, cause that the information in the previous and current (post-partitioned simple) sentences does not include even the information in the current (prepartitioned) sentence. We think it is inadequate, since the antecedents of zero pronouns often appear in the previous (pre-partitioned) sentence. Therefore, it is necessary to extend the range of search for the antecedent to more previous (postpartitioned simple) sentences.

To determine to what extent we should extend the range of search for the antecedent, we make the following investigations and experiment:

- How many simple sentences does a naturally occurring sentence consist of?

- How many sentences from the current sentence do we find the antecedent of a zero pronoun in real discourses?

- How does the accuracy of the zero pronoun resolution change if we vary the range of simple sentences where the antecedent of a zero pronoun is searched?

The first investigation is performed manually, and the result shows that 10,000 sentences of the review articles from the newspaper consist of 24,332 simple sentences. Thercfore, a naturally occurring Japanese sentence can be considered to consist of $2.0-2.5$ simple sentences on average. The second investigation is performed manually on one of the test discourses that are mentioned in the next section, and the result shows that $95 \%$ of the antecedents appcar in the previous or current (pre-partitioned) sentence. This result is consistent with the larger-scale investigation that Fujisawa et al.(Fujisawa et al., 1991) made for the same purpose. Fujisawa's investigation, on 1,087 sentences of the scientific journal and 1,426 sentences of the review articles from the newspaper, showed that $87.6 \%$ of the antecedents appeared in the previous or current sentence and $95.1 \%$ appeared in the previous two sentences or current sentence. The third experiment is performed on two of the test discourses in the next section, by implementing two versions of the centering algorithms that are mentioned in the last section and varying the range of simple sentences where the antecedent of a zoro pronoun is searched from the previous sentence to the previous ten sentences. The experiment shows that the accuracy improves until the previous $2-4$ sentences are searched, but degrades after that.

Totially taking into account, these results, we determine that the antecedents are searched in the previous four simple sentences. Since the antecedent tends to appear in the closer sentence to the zero pronoun, as l'tujisawa's investigation indicates, we determine the following forward center ranking among the $C_{f}$ s of the previous four simple sentences:

$$
C_{f}^{1}>C_{f}^{2}>C_{f}^{3}>C_{f}^{4}
$$

where $C_{f}^{n}$ represents the $C_{f}$ of the n-th simple sentence from the current sentence.

\subsection{Taking into account the information of conjunctive postpositions}

Even if the antecodents are searched in the previous four simple sentences, simple 'partition' approach might not yield good performance, because the information of conjunctive postpositions that are between two adjacent simple sentences is not taken into account. For example, consider the following sentences:

(a) Taro wa issyoukenmei benkyou siteita.

(b) Jiro ga koe wo kake temo, kizukanakatta.

These sentences are partitioned into the following simple sentences:

(a) Taro wa issyoukenmei benkyou siteita. Topic

Taro was studying hard.

(b1)Jiro ga ( $\phi$ ni ) koe wo kake temo, Subj Conj

Although Jiro called out to him,

(b2) $\left(\phi_{1}\right.$ ga $)\left(\phi_{2}\right.$ ni ) kizukanakatta. he did not notice him.

Here $\phi$ represents a zero pronoun. Applying the contering algorithm to these sentences, the process becomes as follows:

$$
\begin{aligned}
& (a): C_{f}=[\text { Taro }], C_{b}=[?]^{3} \\
& (b 1): \phi=\text { Taro, } C_{f}=[\text { Jiro, Taro }], C_{b}= \\
& \text { Taro } \\
& \left(\text { b2) }: \phi_{1}=\text { Jiro, } \phi_{2}=\text { Taro, } C_{f}=\right. \\
& {\left[\text { Jiro, Taro], } C_{b}=\right.\text { Taro }}
\end{aligned}
$$

Therefore, the counter-intuitive interprotation that 'Jiro did not notice Taro' is obtained.

Since two adjacent simple sentences in a complex sentence are combined together by the conjunctive postposition that indicates the relationship between them, using the information of the conjunctive postposition might improve the performance of the zero pronoun resolution.

To clarify how the zero pronoun resolution relies on the information of conjunctive postpositions, we perform the investigation whether the noun phrases with the same grammatical property agree in two adjacent simple sentences that have a conjunctive postposition between them, by extracting sentences with conjunctive postpositions from the review articles in the newspaper and enumerating the agroement and disagreement. The enumeration is performed in cases where both sentences have zero pronouns and only either sentence has

\footnotetext{
${ }^{3}$ The first sentence in a discourse has no $C_{b}$.
} 
a zero pronoun. Twelve main conjunctive postpositions are investigated. The result of the investigation is quite similar to the Yoshimoto's and Minami's investigations(Yoshimoto, 1986; Mirami, 1974) that classify the conjunctive postpositions into three classes:

- Class A: 'nagara' ('while'), 'tari' ('and'), 'tutu' ("while'), 'te' ('and')

If two sentences have a conjunctive postposition of class $A$ between them, the subject noun phrases tend to coincide, in both cases where both sentences have zero pronouns and only cither sentcnce has a zero pronoun.

- Class B: 'temo' ('although'), 'node' ('because'), 'noni' ('although'), 'keredo' ('although'), 'ba' ('if'), 'kara' ('because'), 'to' ('when')

If two sentences have a conjunctive postposition of class $B$ between them, the antecedent tends to be not the subject of the other sentence, in case where only either sentence has the zero pronoun of the subject position. In case where both sentences have zero pronouns, the agreemont/disagreement deponds on the context and does not have any tendency.

- Class C: 'ga' ('but')

The agreement/disagreement depends on the context and does not have any tendency.

¿From this result of the investigation, we determine to apply to the zero pronoun resolution the following heuristics that are concerned with conjunctive postpositions. Since conjunctive postpositions of class $A$ have a strong preference that two subjects in adjacent sentences tend to coincide, instead of the centering algorithm, we use this preference for the zero pronoun resolution in the simple sentence after the conjunctive postpositions of class $\mathrm{A}$, and try to find the antecedents of zero pronouns in the same position of the adjacent sentence, if any. In this case also, the center of the current sentence is computed similarly to the ordinary algorithm, and the antecedent of the zero pronoun becomes the $C_{b}$ of the current sentence.

In case of conjunctive postpositions of class B, the antecedent tends to be not the subject of the other sentence if one of the sentences has the zero pronoun of the subject position. We think this tendency implies that noun phrases in the sentence before the conjunctive postpositions of class $B$ tend to be not the antecedents of zero pronouns in the next sentences. Therefore, wo give these noun phrases the least preforence as tho antecodents, although the zero pronoun resolution is performed by the original centering algorithm.

\footnotetext{
${ }^{4}$ In parentheses, we show the direct translation of conjunctive postpositions into English
}

Since conjunctive postpositions of class $\mathrm{C}$ have no preference for the antecedents of zero pronouns, the zero pronoun resolution is performod as usual.

Consider again the following sentences:

(a) Taro wa issyoukenmei benkyou sitcita. Topic

Taro was studying hard.

(b1)Jiro ga ( $\phi$ ni ) koe wo kake temo, Subj Conj

Although Jiro called out to him,

(b2) $\phi_{1}$ ga ) ( $\phi_{2}$ ni ) ki he did not notice him.

If the original centering algorithm is applied to each sentence uniformly, the counter-intuitive interpretation is obtained, as mentioned above. Taking into account the information of conjunctive postpositions and applying the above heuristics to the points, since (b1) and (b2) have the conjunctive postposition of class B, 'temo' ('although'), between them, the noun phrases in sentence (b1) have the least preference and the order of $C_{f}$ in the sentence (b1) becomes the opposite to the case of the original centering algorithm. Therefore, the antecedents of the zero pronouns in sentence (b2) are identified as follows:

$$
\begin{aligned}
& \text { (a): } C_{f}=[\text { Taro }], C_{b}=[?] \\
& (b 1): \phi=\text { Taro, } C_{f}=[\text { Taro, Jiro }], C_{b}= \\
& \text { Taro } \\
& \text { (b2): } \phi_{1}=\text { Taro, } \phi_{2}=\text { Jiro, } C_{f}= \\
& {[\text { Taro, Jiro }], C_{b}=\text { Taro }}
\end{aligned}
$$

Here this interpretation that "Taro did not notice, Jiro' fits our int,uition.

\section{Experiment and Discussion}

In the last section, we described our zero pronoun resolution method that can handle complex sentences based on the contering theory. It differs from the original centering algorithm in the following two points. After partitioning complex sentences into multiple simple sentences, it searches the antecedents in the previous four simple sentences, instead of only a previous sentence. Secondly, it takes into account the information of conjunctive postpositions that are between two simple sentences, by classifying them into three classes.

In this section, we describe the exporiments that our zero pronoun resolution method is applied to real Japanese discourses, to evaluate the effectiveness. We implement two versions of our zoro pronoun resolution systems which are based on two versions of the centering algorithms that are mentioned in section two respectively, and evaluate the performance by comparing ours with the performance of the original centering algorithms.

As our test discourses, we use 275 (prepartitioned) sentences from five discourses in to tal, which are a review article in the newspaper, a foll-tale, and a novel. Before the experiments, 
Table 1: 'The performance of the systens based on Kameyama's algorithm

\begin{tabular}{|c|c|c|c|c|}
\hline \multirow[t]{2}{*}{ method } & \multirow[t]{2}{*}{ correct, } & \multicolumn{2}{|c|}{ incorrect } & \multirow[t]{2}{*}{ accuracy } \\
\hline & & iin & not in & \\
\hline 1 & 224 & 10 & 80 & $71.3 \%$ \\
\hline 2 & 237 & 46 & 31 & $75 . \overline{5} \%$ \\
\hline 3 & 246 & 36 & 32 & $78.3 \%$ \\
\hline
\end{tabular}

Table 2: 'The performance of the systems based on Walker's algorithm

\begin{tabular}{|l|c|c|c|c|}
\hline method & correct & \multicolumn{2}{|c|}{ incorrect } & accuracy \\
\cline { 2 - 4 } & & in & not in & \\
\hline 1 & 197 & $\frac{31}{72}$ & $-\frac{86}{30}$ & $62.7 \%$ \\
\hline 2 & $21 \frac{2}{67}$ & $\frac{72}{5} \%$ \\
\hline 3 & 230 & 54 & $\frac{30}{73}$ & $\frac{73}{2} \%$ \\
\hline
\end{tabular}

these discourses are automatically partitioned into simple sentences and received structural analysis, and the positions of zero pronouns are antomatically identifed as missing obligatory cases of verbs. Then, the results of this preprocessing are manually corrected. The zero pronoun whose antecedent appears after it, i.e., the catiaphoric one, and the zero pronoun whose antecedent does not appear in the discourse, are outside the scope of this paper. Those zero pronouns are $30 \%$ of all the zero pronoums in our test discourses.

The correct antecedents are minually identilied beforchand against each zoro pronoum, and the? performance is computed based on these answers. The experiments are made on the following thee cases:

1. The original contering algorithm that uses the information of only a previous simple sentence

2. The algorithm that searches the antecedents in the previous four simple sentences

3. 'The algorithm not only saarches the antecedents in the previous four simple sentences, but also takes into account the information of conjunctive postpositions that are between two simple sentencess

'Ihe results of the experimentis on two versions of our systems are shown in lable 1 and 2 , where the columns of 'correct' and 'incorrect' show the numbers of the correct and incorrect, answers that the system outputs respectively, and the columms of 'in' and 'not in' show the numbers of cases where candidates of antecedents in the system include the correct answer and the number of cases whore the system does not have the correct answor as the candiclates, respectively.

Although the original centering algoritims yield the performance of $60-70 \%$, they have many cases where the system cannot get the correct answer as the candidates('not in') and cannot ont, put correct answers. This indicates that there are many cases where the correct antecedents do not appear in the previous sentence, and implies the plausibility of our first modification to the original algorithm. The improvenent of the performance in method 2 also implies the plausibility of our method. Furthermore, taking into account the information of conjunctive postpositions improves the performance by $3 \cdots 6 \%$. Totally, compared with the original centering algorithms, the performance of our method improves by $7-10 \%$.

Since the zero pronoun resolution method that is based on the centering theory uses the results of the zero pronom resolution in previous sontences, "errot-chaining' might occur mauy times. birror-chaning occurs when the identification of a wrong antecedent causes another wrong zoro pronoun resolution successively. In case of our system(nothod 3) based on Kameyama's algorithm, $30.2 \%$ of the incorrect answers are due 10 this erorechaining. There is also the possibility where the correct answers are output because of the wrong zero pronom resolution in the previous sentences. In case of our system(mothod 3 ) based on Kaneyama's algorithrn, only $1.2 \%$ of the correct answers aro due to this 'falso negative.'

As you notice from the above two tables, there exist about 30 cases where the antecedents appoar in more than five sentences from the current simple sentence and annot be found by our ancthod. Wo think these cases should not be hanclled simply by extending the soarch range for the antecedents, but by utilizing the information of global structure of discourses (Gros\% and Sidner, 1986), because the digressive sub-discourse is inserted between the antecedents and the zero pronouns in most of these cases.

Of course, there have been the zero pronom resolution approaches that take into account the inlormation of conjunctive elements(Nakaiwa and Tkehara, 1992; Nakagawa and Nishizawa, 1994; Yoshimoto, 1986; Suri and McCoy, 1994). Bo-cause Nakaiwa's(Nakaiwa and lkohara, 1992) and Nakagawa's(Naliagawa and Nishizawa, 1994) ap)proaches use the information in a restricted domain or too fine-grained grammatical information, wo think they are diflicult to be tuned to the broad coverage zero pronoun resolution system. Fut thermore, Nakagawa's and Yoshimoto's(Yoshimoto, 1986) approachos aro not fully evalualed with roal discourses. Although Nakaiwa's approach yiclds high success rate of $93 \%$, he uses rather small test sets(10)2 sentences from 29 articles), and the input. is restricted to the first paragraphs of newspaper articles.

Suri's work(Suri and Mccoy, 1994) might be: one of the fow works that extend the centerings lianework to hiudle complex sentences, althongh 
she handles only sentences of the form 'SX because SY, and uses Sidner's focusing framework(Sidner, 1983), that is different from the centering theory that our method is based on. Furthermore, the effectiveness of her work is not evaluated with real discourses.

Takada's work(Takada and Doi, 1994) might be the only exception that proposes the zero pronoun resolution method based on the centering theory and evaluates its effectiveness with real discourses. Since he handles not only zero pronouns but also overt pronouns, the exact comparison is difficult, but his approach, that is based on Kameyama's approach, yields the performance of $74.8 \%$ if the results for overt pronouns are excluded. In addition, to handle complex sentences, he adopts the other approach where they are treated as a single unit, and admits that some problems arise because of this approach.

Taking into account the information of conjunctive elements in the pronoun resolution reminds us of the works that use the establishment of coherence relations between clauses for pronoun resolution(Hobbs, 1979; Kehler, 1993). They try to establish coherence relations by the costly inference, while we use only the surface information.

\section{Conclusion}

In this paper, we presented a simple method to handle complex sentences with the centering theory and described our framework that can identify the antecedents of zero pronouns in naturally occurring Japanese discourses. We also presented the evaluation of our framework with real discourses, although the evaluation is not so largescale to assert the effectiveness of our framework. Our simple method yielded the accuracy of $78 \%$ for the zero pronoun resolution.

Since our method that is presented in this paper is based on the centering theory and basically uses only syntactic information, we plan to incorporate the semantic constraints that filter anomalous antecedents for zero pronouns, and take into account the global structure of discourses. We think the preliminary results of our system in this paper are promising since incorporating the information of semantic constraints and the global structure of discourses will improve the performance.

\section{References}

S.E. Brennan, M.W. Friedman, and C.J. Pollard, A Centering Approach to Pronouns, Proc. of the 25th Annual Meeting of the Association for Computational Linguistics, pp.155-162, 1987.

S. Fujisawa, S. Masuyama, and S. Naito, A Basic Study on Ellipsis and Anaphora in Japanese Sentences, IPSJ SIG Notes, Vol. 91, No. 96, in Japanese, 1991.
B.J. Gros\% and C.L. Sidner, Attention, Intentions, and the Structure of Discourse, Computational Linguistics, Volume 12, Number 3, 175-204, 1986.

B.J. Grosz, A.K. Joshi, and S. Weinstein, Centering: A Framework for Modeling the Local Coherence of Discourse, Computational Linguistics, Volume 21, Number 2, pp.203-225, 1995.

J.R. Hobbs, Coherence and Coreference, Cognitive Science, Volume 3, Number 1, pp.67-90, 1979.

M. Kameyama, A Property-Sharing Constraint in Centering, Proc. of the 24th Annual Meeting of the Association for Computational Linguistics, pp.200-206, 1986.

A. Kehler, The Effect of Establishing Coherence in Ellipsis and Anaphora Resolution, Proc. of the 31st Annual Meeting of the Association for Computational Linguistics, pp.62-69, 1993.

F. Minami, Gendai Nihongo no Kouzou, Taishuukan, Tokyo, in Japanese, 1974.

H. Nakagawa and S. Nishizawa, Semantics of Complex Sentences in Japanese, Proc. of the 15th International Conference on Computational Linguistics, pp.679-685, 1994.

H. Nakaiwa and S. Tkehara, Zero Pronoun Resolution in a Japanese to English Machine Translation System by using Verbal Semantic Attributes, Proc. of the 3rd Conference on Applied Natural Language Processing, pp.201-208, 1992.

C.L. Sidner, Focusing in the Comprehension of Definite Anaphora, In M. Brady and R.C. Berwick, editors, Computational Models of Discourse, MIT Press, pp.267-330, 1983.

L.Z. Suri and K.F. McCoy, RAFT/RAPR and Centering: A Comparison and Discussion of Problems Related to Processing Complex Sentences, Computational Linguistics, Volume20, Number 2, pp.301-317, 1994.

S. Takada and N. Doi, Centering in Japanese: A Step 'Towards Better Interpretation of Pronouns and Zero-Pronouns, Proc. of the 15th International Conference on Computational Linguistics, pp.1151-1156, 1994.

M.A. Walker, M. Iida, and S. Cote, Japanese Discourse and the Process of Centering, Computational Linguistics, Volume 20, Number 2, pp.193-232, 1994.

K. Yoshimoto, Study of Japanese Zero Pronouns in Discourse Processing, IPSJ SIG Notes, Vol. 86, No. 52, in Japanese, 1986. 